\title{
Face Detection Technology Based on Robot Vision
}

\author{
Guxiong Li \\ Guangzhou College of South China University of Technology , \\ 510800,Guangzhou
}

\begin{abstract}
One being developed automatic sweep robot, need to estimate if anyone is on a certain range of road ahead then automatically adjust running speed, in order to ensure work efficiency and operation safety. This paper proposed a method using face detection to predict the data of image sensor. The experimental results show that, the proposed algorithm is practical and reliable, and good outcome have been achieved in the application of instruction robot.
\end{abstract}

Keywords: face detection; gray integral; skin color model; robot vision

\section{Introduction}

Nowadays, automatic sweep robot makes people free from the labor, greatly improves the people's living standard. Man has more energy to engage in meaningful activities or work. One being developed automatic sweep robot, need to estimate if anyone is on a certain range of road ahead. If nobody here, robot will be in cleaning operations at $30 \mathrm{~km} / \mathrm{h}$ speed. If somebody there, ECU issued a directive to make vehicle deceleration. Speed of automatic sweep robot reduces from $30 \mathrm{~km} / \mathrm{h}$ to $10 \mathrm{~km} / \mathrm{h}$, to ensure no personal injury. ECU connects the speaker sends out music to warn pedestrians.

After the pedestrian pass, there is no face detection in image data. ECU issued a directive to make vehicle accelerate to $30 \mathrm{~km} / \mathrm{h}$, in order to obtain a higher efficiency. ECU disconnects the speaker to ensure no noise pollution. In this thesis, we use face detection to mine data, forecast if anyone is on a certain range of road ahead successfully.

\section{Summary of face detection}

Face detection, a key technology of face information processing, is the subject of pattern recognition and computer vision. Its nature is characteristic for the face region from other region $[1,2]$.

The basic character of face can be summarized as color feature (skin color, hair color, lip color, gray), geometric characteristics (Contour, facial features, symmetry), statistical characteristics (mean, variance, correlation coefficient, histogram, mosaic features). Face detection algorithms can be divided into two categories: knowledge based method and statistic based method. To summarize characteristics of face region distinguishes from the non-face region, the method based on knowledge is observation with the naked eye by the people. According to different face feature, they are template matching method, method based on the complexion model, mosaic method, face geometry method.

Based on the statistical method takes the human face region as a kind of mode, using of a large number of "face", "non-face" samples for training, in order to construct classifier. There are characteristic face method, artificial neural network, support vector machine.

In this thesis, we use gray integral and color model combination method to detect face. Firstly, we use gray integral method to realize Preliminary location of the face. Use segmentation method for skin color region of YIQ color model to achieve further location of the face. Accurate face detection comes true ultimately. 


\section{Gray-level projection theory}

After research, we can catch sight of that the face region will make the vertical gray-level projection form a peak with certain breadth. Left and right boundary of this convex peak represents the boundary of face roughly. We focus on the horizontal gray projection curve of the face region image. For the curve, point of the first minimum value corresponds to the man's head, because the low gray of hair contributes to low ebb of horizontal projection curve. Point of the maximum value corresponds to the man's forehead, and point of the second maximum value corresponds to the man's nose [3].

Edge is the boundary where the image gray changes dramatically. Changes situation of gray scale can be reflected by gradient of image gray distribution. For a continuous image, such as $f(x, y)$,

gradient of position $(x, y)$ can be expressed as a vector :

$$
\Delta f(x, y)=[\partial f / \partial x, \partial f / \partial y]^{T}=\left[f_{x} f_{y}\right]^{T}
$$

This vector contains the changes information of local gray, its range is:

$$
e(x, y)=\sqrt{f_{x}^{2}(x, y)+f_{y}^{2}(x, y)}
$$

We can also use the absolute value of partial derivative $f_{x}, f_{y}$ as the gray variation amplitude

$$
e(x, y)=\left|f_{x}(x, y)\right|+\left|f_{y}(x, y)\right|
$$

For the vertical edge, $f_{x}$, the partial derivative of vertical direction, is approximate zero. The boundary of cheek is longer than the vertical edge of anyother facial features, so the sum of difference of horizontal gray level corresponding to the boundary of cheek must be the biggest. In the integral projection of vertical direction of gray difference, there are two maximum value at both ends, which are respectively corresponding to the two boundary lines of cheek. Assuming that it spans two pixel width, arithmetic for detection of gray difference of Approximate vertical edge is:

$$
g(x, y)=|f(x, y)-f(x, y-1)|+|f(x, y)-f(x, y+1)|
$$

The algorithm can be described as follows:For each column, calculate gray difference for each pixel and two adjacent pixel. Then, add gray difference of each pixel of a list togethe as $v(y)$. From the first column on the left side to the $1 / 3$ width of the image, find the largest $v(y)$, where $\mathrm{y}$ corresponds to the left boundary (left cheek).We can figure out the right boundary (right cheek) in the same method.

The sum of difference of gray level corresponding to the vertical direction of the hair hanging down may be the biggest, too. We don't expect to interfere with this situation. So, we carry on gray difference calculation and summation of $f(x, y)$ when $f(x, y+1)$ is larger than $f(x, y-1)$.

\section{Segmentation of skin color region based on YIQ model}

Skin color is an important information of face. Skin color is relatively stable, can distinguish itself from the most background color. It does not depend on the face Details, is available to revolving image, varied facial expression,etc. Then, use the area feature of geometric and gray to verify whether the face is, and to exclude other objects in similar color[4].

The idea for segmentation of skin color region is as follows: For the input image,transform the RGB model into YIQ model Firstly. Find out the elliptic region based on YIQ model. Choose the right threshold value to carry out images binaryzation. Then, it could be in dnoising processing. Carry through closed operation based on morphological image processing (first expansion then corrosion). We carry on image opptimization processing of skin color region,based on the length $\&$ width ratio theory and effective area theory. 


\section{Color model transformation}

Clustering of skin color based on YIQ model is more obvious than Clustering of skin color based on RGB model, so we employ elliptic region approache based on YIQ model. Y express luminance or brightness of color. I and Q indicate chromiance of color, or say saturation of image color[5]. The relationship between YIQ model and RGB model is follow:

$$
\left[\begin{array}{l}
Y \\
I \\
Q
\end{array}\right]=\left[\begin{array}{ccc}
0.299 & 0.587 & 0.114 \\
0.596 & -0.274 & -0.322 \\
0.211 & -0.523 & 0.312
\end{array}\right]\left[\begin{array}{l}
R \\
G \\
B
\end{array}\right]
$$

If pixel p satisfy the inequality (6), $p$ is skin clore pixe. Or else, $p$ is non-skin clore pixe.

$$
\begin{aligned}
& \frac{\left(I_{p}-m\right)^{2}}{r^{2}}-\frac{Q_{p}{ }^{2}}{q^{2}} \leq 1 \\
& I_{p} \text { and } Q_{p} \text { indicate the I component and } \mathrm{Q}
\end{aligned}
$$
component corresponding to $\mathrm{P}$ point. Then $\mathrm{r}$ and $\mathrm{q}$ show the scope of I component and scope of $\mathrm{Q}$ component corresponding to $\mathrm{P}$ point, $\mathrm{m}$ say the mean value of I componen.

\section{Morphological operation}

There are some noises in the background after image binaryzation, which make the subsequent operations more complex. So we utilize morphological filter to remove noise on the face candidate regions.

Mathematical morphology comprises corrosion, expansion, open operation, close operation, etc. We could wield these operators and their combination to execute analysis and processing for the shape and structure of image $[6,7]$.

Open operation: first corrosion, and then expansion. It can not only eliminate small object, separate object in fine point, smooth boundaries of larger object, but also not change area distinctly.

Its definiens is:

$$
B \bullet S=(B \otimes S) \oplus S
$$

Close operation: first expansion, and then corrosion. It can not only fill in internal fine model of object, connect adjacent objects, smooth boundaries, but also not change area distinctly. Its circumscription is:

$$
B \bullet S=(B \oplus S) \otimes S
$$

Morphological processing makes it possible to narrow the face candidate region and improve the detection speed. It fills in small hole of skin color region simultaneously. Those exiguous cavities can be mistaken for face organ sometimes.

\section{Optimization of skin color region}

We implement regional optimization based on hight-width ratio and area occupancy rate theoretics.

Research shows that the golden ratio of height and width is

$$
R_{h w}=\frac{1+\sqrt{5}}{2}
$$

Theoretically, it is likely to be the face region if the hight-width ratio of a candidate region is between 0.8 and 1.6. Considering the influence of naked neck, the hight-width ratio intercalate value from 0.8 to 1.8 in this text. Image region which is beyond this range do not be considered[8]. Therefore we can suppress the interference of hand, arm and anyother limbs and trunk which ar exposed outside effectively to achieve high precision.

\section{Experimental result}

The capture images by cameras are shown in Figure 1. 


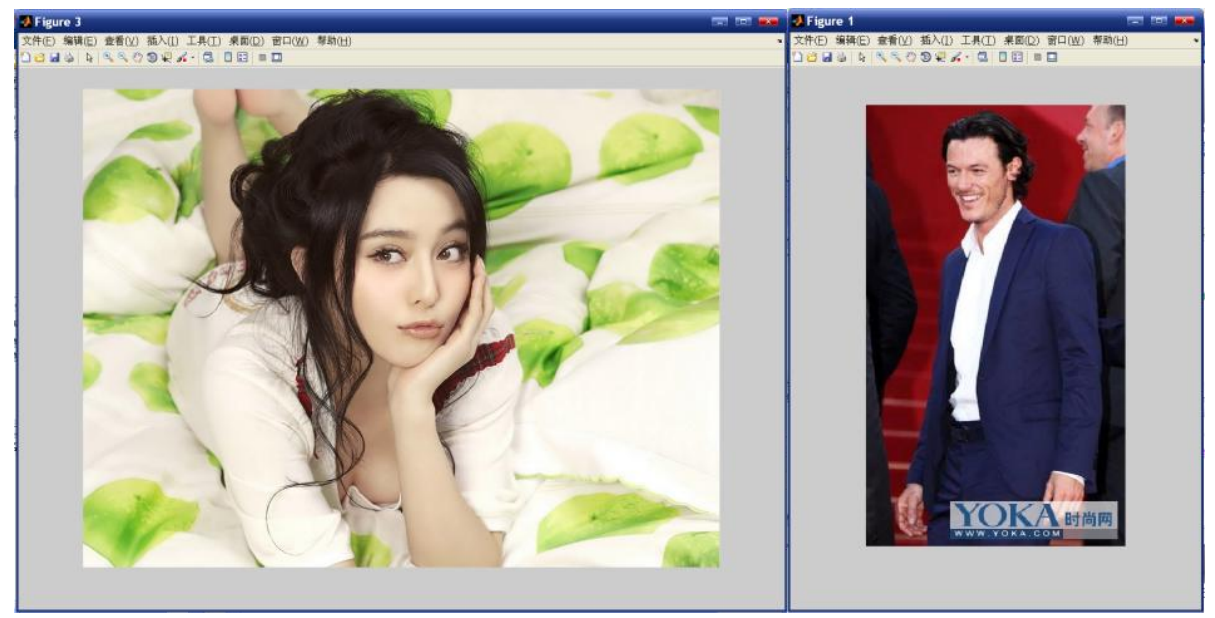

Fig.1 the capture images

Face detection images are shown in Figure 2. It indicates that the program achieved the intended function.

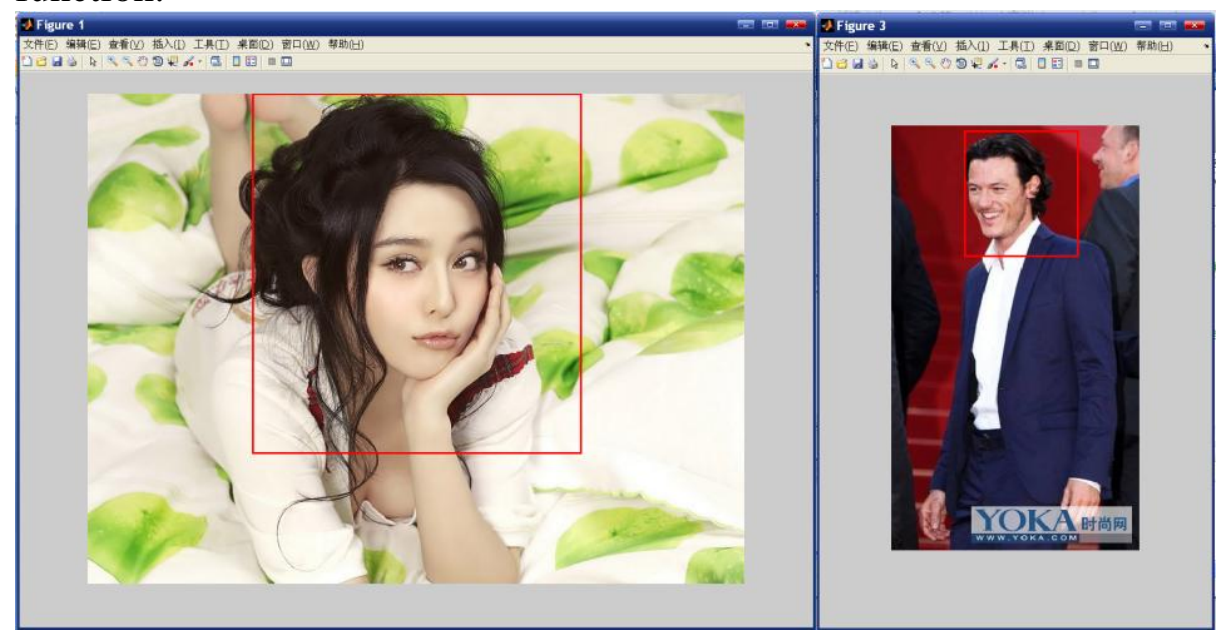

Fig.2 Face detection images

\section{Conclusions}

In this paper, we propose a face detection method which is applied in automatic sweep robot. The progressiveness of research reflects in the combination of gray integral method and color model method to detect face accurately. Performance in the appliance shows that the method is of high accuracy and trustworthy. The deficiency is that it can only enable face detection of single person, which points out the direction for the improvement of our future work.

\section{References}

[1] Ai Haizhou, Liang Luhong. Face Detection based on Skin Color and Template[J]. Journal of Software. 2014,12 (12): 17-25.

[2] $\mathrm{Su}$ Jianbo, $\mathrm{Xu}$ Bo. Face Recognition and Speech Recognition [M]. Shanghai: Shanghai Jiao Tong University Press. 2013.

[3] Wang Hongman. Extraction and Recognition of Algebraic Feature and Geometric Feature of Face [D]. Dalian: Dalian University of Technology. 2014. 
[4] Bian Zhaoqi, Zhang Xuegong.Pattern Recognition $[\mathrm{M}]$. Beijing: Tsinghua University Press, 2014.

[5] Zhu Junqing, Wang Linquan, Ge yuan. Fast Face Detection based on Template Matching[J]. Computer Engineering. 2013, 28 (9): 77-81.

[6] Li Huasheng, Yang Hua, Yuan Baozong. Feature Extraction of Face Recognition Systems [J]. Journal of Northern Jiaotong University. 2014, 25: (2).

[7] Lin Fuyan, Liu Qiaojing, Li Xingsen. Localization and Extraction of Face Feature [J]. Application of Electronic Technology. 2013, 7:17-21.

[8] Guo Rui Zhang Shufen Wang Xiaoya. Research on Extraction of Face Recognition Feature and

Similarity Matching Method [J]. Computer Engineering. 2014,32 (11):25-27.
[9] Michael J. Swain,Dana H. Ballard. Color indexing $[\mathrm{J}]$. International Journal of Computer Vision ,2014 (1): 7-9.

[10] Fikriye ztürk,Figen zen.A New License Plate Recognition System Based onProbabilistic Neural Networks[D]. Procedia Technology . 2014: 8-15

[11] Elliamn D G and Lancaster I T. A review of segmentation and contextual analysis techniques for text recognition[M]. Pattern Recognition. 2013: 20-23

[12] Androutsos D.A Novel Vector-Based Approach to Color Image Retrieval Using a Vector Angular-Based Distance Measure[J]. Computer Vision and Image Understanding,2014: 25-30.

[13] Kdhan S and Pavlidis T.On the recogition of printed characters Of any font and size $[\mathrm{J}]$. IEEE Trans. Pattern Anal.Mach.Intell , 2014: 30-34. 